\title{
Energy Spread and Emittance Simulation for RISP RFQ Cooler
}

\author{
Young-Ho Park ${ }^{\mathrm{a}}$, Ju Hahn Lee, Won Joo Hwang, Gi Dong Kim, Hyung-Joo Woo, Yong Kyun Kim \\ Rare Isotope Science Project, Institute for Basic Science, Daejeon 305-811, Republic of Korea
}

\begin{abstract}
RFQ cooler of RAON facility is a device to enhance the quality of ion beam. It is to secure the resolving power of high resolution mass separator by reducing the energy spread and the emittance of ion beam. Key components of this device are deceleration optics for ions with kinetic energy of up to $40 \mathrm{keV}$, segmented RFQ electrodes for trapping and cooling, and extraction optics for reacceleration. Using SIMION simulation code, we could test the device and characterize the output beam quality. Output emittance of less than $3 \pi \mathrm{mm}$ mrad and energy spread of less than $1 \mathrm{eV}$ were obtained in this simulation.
\end{abstract}

\section{Introduction}

Ions with well-defined position and momentum are inevitable for precision measurement and separation. RFQ cooler [1] is to prepare ion beam with precise physical properties concerning emittance, energy spread, and temporal spread etc. As ions are thermally cooled by buffer gas collision in a radio frequency quadrupole trap, they come to have better beam quality than the ones that were originally generated from an ion source. In RAON facility [2], an RFQ cooler will be installed in between pre-separator of ion source and high resolution mass separator of low energy beam line. The target specifications of this device are as follows: output emittance of $3 \pi \mathrm{mm} \mathrm{mrad}$, energy spread of less than $1 \mathrm{eV}$, and transmission efficiency of more than $60 \%$ in DC mode operation. We started our study with a design similar to TITAN RFQ cooler [3]. The space charge effect from the Coulomb repulsion between individual ions was not taken into account in this study. We report the performance of our RFQ cooler based on the calculation from SIMION code [4].

\section{RFQ Cooler Design}

RFQ cooler is composed of injection optics, segmented RFQ, and extraction optics as depicted in Figure 1. Passing through the injection optics, the ions lose their kinetic energy down to $\sim 100 \mathrm{eV}$. Reduction of beam energy delivers enough time for the thermalization process during the passage through buffer gas region. An einzel lens is used in the injection part to effectively transport ions into RFQ area. In order to apply confining potential along the beam direction, RFQ electrodes are segmented in 25 segments with length of $40 \mathrm{~mm}$ or $20 \mathrm{~mm}$. Shorter ones in the end part of the RFQ are to form narrower potential well for the bunch mode operation. The radial distance between

\footnotetext{
${ }^{\text {a }}$ Corresponding author: yhpark@ibs.re.kr
} 
opposite electrodes of RFQ is $20 \mathrm{~mm}$. Drag force model in the simulation works fine and fast but the ultimate temperature goes to zero as the cooling time increases. It means that we cannot obtain the actual energy spread using drag force model. Therefore, we applied hard-sphere collision model instead for the realistic simulation. The $77 \mathrm{~cm}$-long trap region are filled with He buffer gas of which the pressure is about $10^{-2}$ mbar. Diaphragms with small hole are located at the entrance and the exit of RFQ region, where high vacuum condition is required for the efficient transmission and minimal perturbation during reacceleration of the ions.

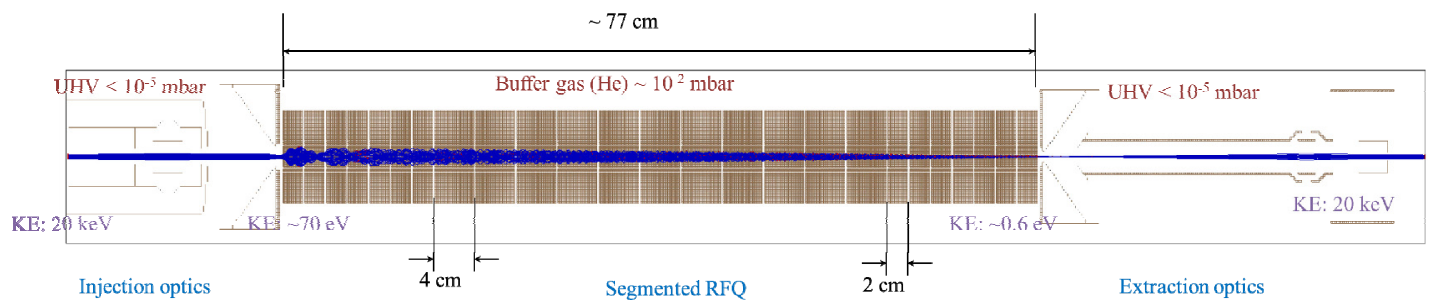

Figure 1. Dimension and simulation parameters for RFQ cooler.

\section{Simulation using SIMION code}

One thousand ${ }^{132} \mathrm{Sn}^{+}$ions were injected into RFQ-cooler in the simulation of DC mode operation. The frequency and the amplitude of $\mathrm{rf}$ field were set to $1 \mathrm{MHz}$ and $400 \mathrm{~V}$ respectively. We generated ions with initial emittance $\left(\varepsilon_{86.4 \%}\right)$ of $30 \pi \mathrm{mm}$ mrad and energy spread of $10 \mathrm{eV}$ (FWHM) using Gaussian random numbers. Pressure inside the RFQ region was set to $5.3 \times 10^{-3}$ mbar while that of the other region was set to $5 \times 10^{-6}$ mbar. We assumed the temperature of the He buffer gas remained constant during the thermalization process. Final emittance at the end of RFQ-cooler was $1.8 \pi \mathrm{mm}$ mrad. Energy spread was reduced down to $0.6 \mathrm{eV}$ (FWHM). Figure 2 shows the transmission efficiency against the vacuum pressure outside the RFQ region. As it is shown below, more ions are lost in the extraction part than in injection area. It reflects that vacuum condition or the differential pumping scheme at the starting point of reacceleration is critical for the final transmission efficiency. Vacuum pressure outside the buffer gas region should be below $1.5 \times 10^{-5}$ mbar in order to obtain transmission efficiency of more than $60 \%$. We expect that a vacuum pump with volume flow rate of more than $800 \mathrm{~L} / \mathrm{s}$ will suffice this requirement. From above simulation, we could assure that this design of RFQ cooler satisfies our target specifications for DC mode operation.

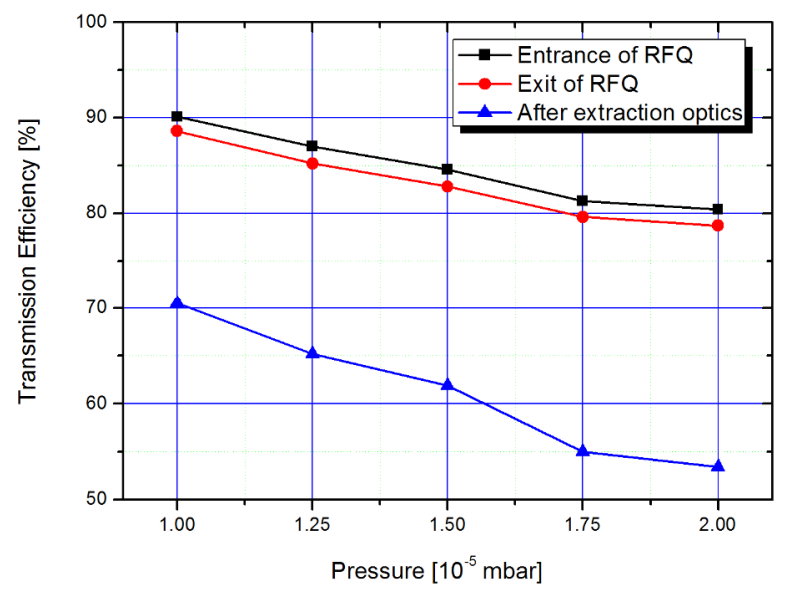

Figure 2. Transmission efficiency against the vacuum pressure outside RFQ region. 
For the bunch mode operation, we set dc voltages to the segmented electrodes to form the potential well along the beam direction whereas the vacuum conditions and the rf voltages are the same as for DC mode operation. Linearly decreasing voltages are set to the electrodes from the first to the 23rd. The last three electrodes are used to form the potential well to bunch ions or the potential ramp to push ions. Adequate timing sequences are arranged for loading, cooling, and pushing the ions. Figure 3 shows the energy spread of the ion bunch when it is being extracted from the capture position. The dashed vertical line represents the position of diaphragm for differential pumping. The lower part of Figure 3 shows the potential array for RFQ and the extraction electrode that are scaled to match with the dimension of $\mathrm{x}$-axis of upper graph. As can be seen from the graph, the growth of the energy spread occurs only in the buffer gas region. That is because the colliding buffer gas atoms drag the ions randomly which are being accelerated during the extraction. Therefore if we can reduce the distance between the capture position and the location of the diaphragm, we will be able to reduce the energy spread of the ion bunch.

Furthermore, the buffer gas pressure of the acceleration region after capture position is thought to be critical for final energy spread of the ion bunch. In order to take account of the precise pressure around the diaphragm aperture, we used Molflow + code [5] to calculate the vacuum pressure. The aperture diameters of the injection and extraction diaphragms are $10 \mathrm{~mm}$ and $5 \mathrm{~mm}$ respectively. Logarithm of the pressure is well fitted to exponentially decaying function along the beam path outside the buffer gas region. A few $\mathrm{cm}$ is needed for the vacuum pressure at the extraction region to be lowered down to the ultimate pressure. We applied this pressure distribution in the SIMION simulation code for the calculation of bunch and extraction of the ions. We shortened the length of the last electrode to $5 \mathrm{~mm}$ in order to minimize the energy broadening in the buffer gas area. The potential array of the extraction electrodes were built in finer grid in order to reduce the calculation error of the potential value around the acceleration region, where the solution of the Laplace equation tends to be erratic because of the large voltage change between the RFQ electrode and the drift tube.

Changes of energy spread, emittance and transmission efficiency against the location of diaphragm are shown in Figure 4. There is an optimal position of the diaphragm that minimizes energy spread and maximizes transmission efficiency. The optimal position is around the capture position (24th electrode). The emittance is not much degraded in that position, however. At optimal conditions, we obtained the energy spread of $0.6 \mathrm{eV}$ (FWHM) and the emittance of $1.7 \pi \mathrm{mm}$ mrad that are similar to those in DC mode operation.

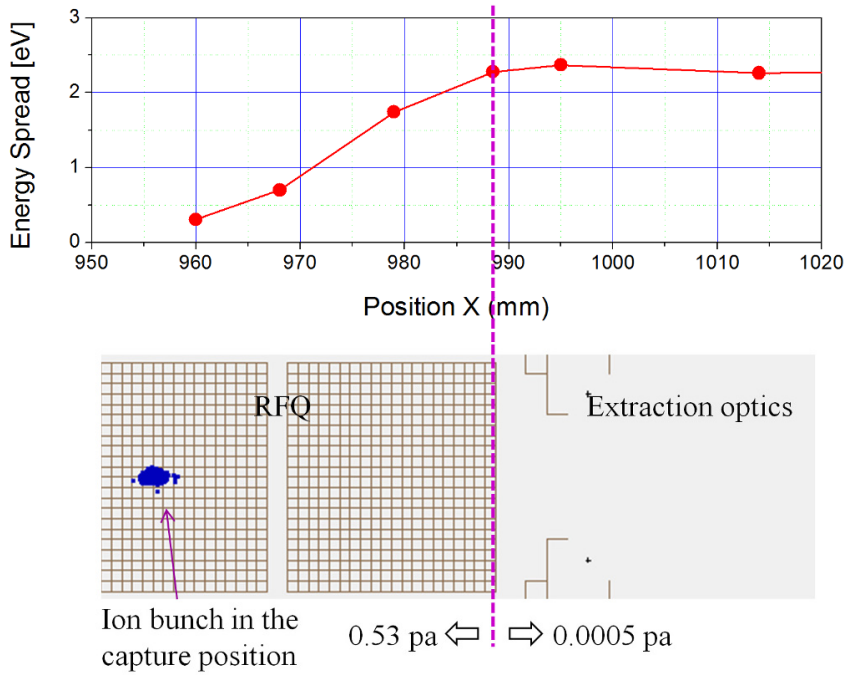

Figure 3. Enegy spread of the ion bunch during extraction from the RFQ. 

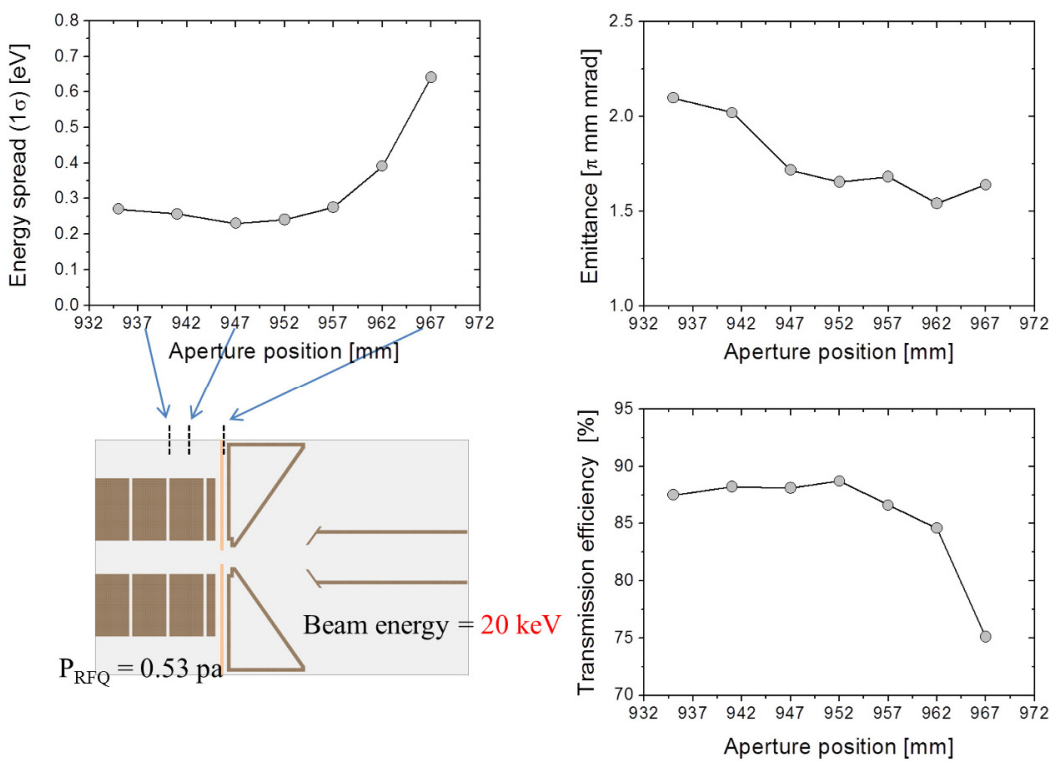

Figure 4. Changes of the beam quality against the position of the diaphragm for differential pumping.

\section{Summary and discussion}

As a tool for beam quality enhancement, an RFQ cooler is designed and tested by an ion optics simulation software i.e. SIMION before it is manufactured and installed. Our concerns were the final emittance, energy spread, and transmission efficiency according to its operating conditions. Although the energy spread of ions in thermal equilibrium at room temperature is less than $0.1 \mathrm{eV}$, the extraction and reacceleration process for the ions inevitably brings the uncertainty of energy and of position etc. For the bunch mode operation, we tuned the position of diaphragm to minimize energy spread using realistic pressure distribution along the beam path. Our target specifications are fulfilled in the design tested in this study. We are convinced that there are important parameters for the performance of RFQ cooler such as vacuum condition in the extraction region, position of the diaphragm, and voltage gradient in DC mode operation etc. The performance of the designed RFQ cooler is summarized in Table 1.

Table 1. Performance of the RFQ cooler in this study.

\begin{tabular}{|c|c|c|c|}
\hline Operation mode & Emittance & Energy Spread & Efficiency \\
\hline DC mode & $1.8 \pi \mathrm{mm} \mathrm{mrad}$ & $0.6 \mathrm{eV}$ & $>60 \%$. \\
\hline Bunch mode & $1.7 \pi \mathrm{mm} \mathrm{mrad}$ & $0.6 \mathrm{eV}$ & - \\
\hline
\end{tabular}

\section{References}

1. R. B. Moore, O. Gianfrancesco, R. Lumbo, S. Schwarz, "The use of high RFQ fields to manipulate ions," Int. J. Mass Spectrom. 251 (2-3) 190-197 (2006).

2. K. Tshoo et al., "Experimental systems overview of the Rare Isotope Science Project in Korea," Nucl. Instr. Meth. B (2013), http://dx.doi.org/10.1016/j.nimb.2013.05.058.

3. M. Smith, L. Blomeley, P. Delheij, J. Dilling, "First tests of the TITAN digital RFQ beam cooler and buncher," Hyperfine Interactions 173 (1) 171-180 (2006).

4. A charged particle optics simulation software (http://simion.com).

5. A Monte-Carlo simulation package developed at CERN (http://test-molflow.web.cern.ch). 